\title{
Glycyl-prolyl-p-nitroanilidase (GPNAase) Activity in Cardiovascular Diseases
}

\author{
Kouichi Ogawa, M.D. and Toshiharu Nagatsu, M.D.
}

\begin{abstract}
SUMmaRY
We have studied serum glycyl-prolyl-p-nitroanilidase (GPNAase) activity and its distribution in various parts of the body in patients with acute myocardial infarction, chronic ischemic heart disease, valvular heart disease with or without congestive heart failure and essential hypertension. Serum GPNAase activity in patients with acute myocardial infarction was significantly lower as long as 12 days after the onset as compared with normal controls. The serum enzyme activity in patients with congestive heart failure was also significantly lower than that of controls and there was a tendency of its gradual decrease with the progress of the disease. There was no significant difference between the activity in control group and that in age-matched patients with essential hypertension. There was no significant change of the activity in patients with chronic ischemic heart disease. The GPNAase activities in sera obtained from various parts of the body by cardiac catheterization were essentially similar.
\end{abstract}

\section{Additional Indexing Words:}

Acute myocardial infarction Ghronic ischemic hcart disease Valvular Heart disease Congestive heart disease Essential hypertension

TN 1966, Hopsu-Havu and Glenner ${ }^{1 /}$ discovered a new dipeptidyl-aminopeptidase (X-prolyl dipeptidyl-amino peptidase), which cleaved N-terminal X-proline from peptides, by using a newly synthesized chromogenic substrate, glycylproline $\beta$-naphthylamide. This enzyme was designated glycylproline $\beta$-naphthylamidase. We found glycylproline $\beta$-naphthylamidase activity in sera of various animals and in the human ${ }^{2,3)}$ and showed that the activity of the enzyme in serum was abnormally high in patients with hepatobiliary diseases, and was decreased in patients with gastric cancer, ${ }^{4}$ ) In chronic carbon tetrachloride intoxication of rats, the activity of the enzyme increased in serum and decreased in liver, suggesting that the enzyme was

From the Second Department of Internal Medicine, Nagoya University School of Medicine and Laboratory of Cell Physiology, Department of Life Chemistry, Graduate School at Nagatsuta, Tokyo Institute of Technology.

Address for reprint: Kouichi Ogawa, M.D., Second Department of Internal Medicine, Nagoya University School of Medicine, 65 Tsuruma-cho, Showa-ku, Nagoya 466, Japan.

Received for publication January 27, 1979.

Manuscript revised February 23, 1979. 
released from the liver in hepatic injury. ${ }^{5)}$ We synthesized various dipeptide $p$-nitroanilides having a sequence of X-proline $p$-nitroanilide (X: Gly, Ala, Lys, Arg, Glu, and Asp) as new chromogenic substrates to develop a new spectrophotometric assay of the activity of X-prolyl dipeptidyl-aminopeptidase and showed that a pure enzyme from the human submaxillary gland ${ }^{6}$ ) hydrolyzed various $\mathrm{X}$-proline $p$-nitroanilides to produce $\mathrm{X}$-proline and $p$-nitroaniline. Glycylproline $p$-nitroanilide was shown to be the best substrate as compared with $p$-nitroanilides of Ala-Pro, Lys-Pro, Arg-Pro, Glu-Pro, and Asp-Pro. ${ }^{7}$ We chose $p$-nitroanilides of $\mathrm{X}$-proline rather than the $\beta$-naphthylamides, since $\beta$-naphthylamide was carcinogenic and not suitable for routine use for the assay of the enzyme. In a previous paper, ${ }^{9}$ ) we reported that glycylproline $p$-nitroanilidase (GPNAase) activity in serum from male patients with essential hypertension was significantly higher than that from normotensive healthy subjects. In this report we have further examined the serum enzyme activity in patients with various cardiovascular diseases.

\section{Materials and Methods}

Glycyl-L-proline $p$-nitroanilide tosylate was newly synthesized as reported previously. ${ }^{7)} \mathrm{X}$-prolyl dipeptidyl-aminopeptidase activity in serum was assayed with glycyl-L-proline $p$-nitroanilide tosylate as substrate by a modification of previously reported continuous direct method $^{7)}$ with use of the LKB 8600 Reactor Rate Analyzer. $\left.{ }^{9}\right)$ The reaction mixture ( $1.15 \mathrm{ml}$ ) contained (final concentrations) $150 \mathrm{mM}$ Tris-HCl Buffer (pH 7.9), $60 \mathrm{mM}$ glycylglycite, $1.63 \mathrm{mM}$ glycylproline $p$-nitroanilide tosylate, and it also contained $50 \mu \mathrm{l}$ of human serum. The reaction was done with the Analyzer at $37^{\circ} \mathrm{C}$, and $p$-nitroanilide liberated by the enzyme reaction was assayed based on the absorbance increased at $410 \mathrm{~nm}$. One unit of the enzyme activity was defined as the amount of enzyme to form $1 \mu \mathrm{M}$ of $p$-nitroanilide per minute (IUB unit, I.U.).

Out of 22 patients with valvular heart disease studied ( 6 patients with mitral stenosis, 6 with combined mitral stenosis and regurgitation, 4 with combined mitral and aortic regurgitation, 4 with aortic regurgitation, and 2 with mitral regurgitation; mean age 43 years, 14 males and 8 females), 10 patients were in NYHA class I (mean age 43 years). Other patients studied were 8 with congestive heart failure, in NYHA class II (mean age 43.6 years), 6 with congestive heart failure, in NYHA class III-IV (mean age 37 years at the time of the study), 9 with acute myocardial infarction (mean age 65 years, 7 males and 2 females), 10 with chronic ischemic heart disease (mean age 59 years, 8 male and 2 females), and 105 with essential hypertension ( 42 males, mean age 49 years; and 63 females, mean age 54 years).

Human blood samples were collected by venipuncture. Thirteen patients (mostly with mitral stenosis) were studied by cardiac catheterization and blood was taken from inferior vena cava, pulmonary artery and femoral artery. The serum was separated by centrifugation at $3,000 \mathrm{rpm}$ for $15 \mathrm{~min}$. The findings obtained in this study are expressed as the mean for the group \pm standard error of 
the mean and were analyzed by Student's " $t$ "-test.

\section{Results}

Fig. 1 shows the activities of serum GPNAase in patients with valvular heart disease with or without congestive heart failure. Mean normal serum GPNAase activity of 1425 normal volunteers was $67.9 \pm 0.3$ I.U. Mean

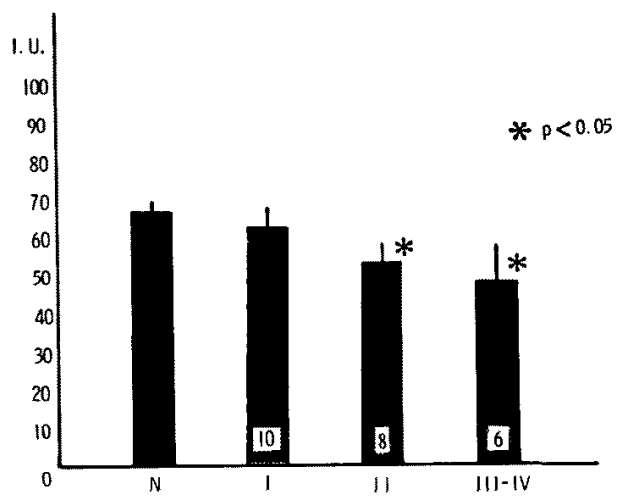

Fig. 1. GPNAase activities in patients with valvular heart disease with or without congestive heart failure.

NYHA class I group: 3 mitral stenosis, 3 combined mitral stenosis and regurgitation, 2 aortic regurgitation and 2 combined mitral and aortic regurgitation.

NYHA class II group: 2 mitral stenosis, 2 combined mitral stenosis and regurgitation, 2 combined mitral and aortic regurgitation, and 2 mitral regurgitation.

NYHA class III-IV group: 2 aortic regurgitation, 2 mitral regurgitation, and 2 combined mitral stenosis and regurgitation.

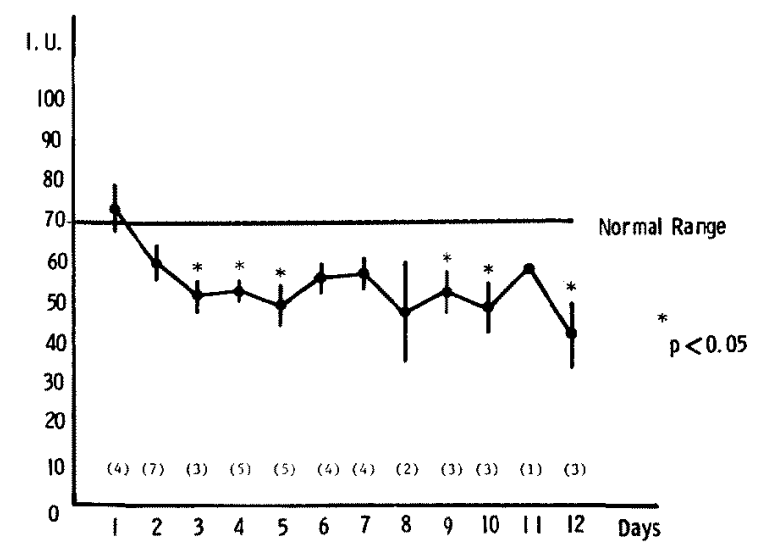

Fig. 2. GPNAase activities after myocardial infarction. Number of patients in parentheses. 
serum GPNAase activity of 10 patients with valvular heart disease in NYHA class I was $66.4 \pm 4.8$ I.U., that of 8 patients in NYHA class II $53.3 \pm 5.9$ I.U., and that of 6 patients in class III-IV $48.2 \pm 8.8$ I.U. The difference of GPNAase activities between the normal group and patients with congestive heart failure in NYHA class II or class III-IV was significant $(p<0.05)$. The difference of GPNAase activities between patients in class $I$ and that in class II or class III-IV was not significant $(p<0.1)$. But there was a tendency of decrease of GPNAase activity with the progress of congestive heart failure.

Fig. 2 shows serial GPNAase activities after acute myocardial infarction. The activity of GPNAase remained to be decreased for 12 days after the onset. The decreases in the activity were significant $(p<0.05)$ at the $3 \mathrm{rd}, 4$ th, 5th, 9 th, 10th, and 12th days after acute myocardial infarction as compared with that of the 1st day. The level had never returned to normal by 12 days after the infarction.

Table I shows GPNAase activity in patients with chronic ischemic heart disease. Mean serum GPNAase activity in 10 patients with chronic ischemic heart disease was $66.6 \pm 5.2$ I.U., and no significant difference from the

Table I. GPNAase Activity in Patients with Chronic Ischemic Heart disease

\begin{tabular}{l|c|c}
\hline & Normal & IHD \\
\hline GPNAase Activity I.U. & $67.9 \pm 0.25$ & $66.6 \pm 5.2$ \\
No. & 1425 & 10 \\
IHD $=$ ischemic heart disease. All patients have experienced angina attacks.
\end{tabular}

Table II. Serum GPNAase Activity in Patients with Essential Hypertension

\begin{tabular}{ll|c|c|c}
\hline \multicolumn{2}{r|}{ Control } & & \multicolumn{2}{|c}{ Hypenrtesives } \\
\hline \multirow{2}{*}{ Male } & No. & 1227 & Without Drugs & With Drugs \\
& B.P. & & 11 & 11 \\
& GPNAase & $68.8 \pm 0.27$ & $172.7 \pm 7.4 / 107.1 \pm 4.1$ & $142.4 \pm 8.1 / 76.7 \pm 3.2$ \\
& Age & $39.9 \pm 0.9$ & $42.5 \pm 3.9$ & $70.8 \pm 3.4$ \\
Female & No. & 198 & 19 & $50.9 \pm 3.9$ \\
& B.P. & & $181.3 \pm 4.4 / 100.8 \pm 2.9$ & $160.9 \pm 4.8 / 93.7 \pm 8.0$ \\
& GPNAase & $62.4 \pm 0.62$ & $60.2 \pm 2.5$ & $71.6 \pm 3.5 *$ \\
& Age & $28.6 \pm 0.82$ & $55.6 \pm 2.5$ & $54.3 \pm 2.0$
\end{tabular}

* p less than 0.02 as compared with controls. Drugs $=$ mostly diuretics, $\beta$-blockers and vasodilators; B.P.=blood pressure, $\mathrm{mmHg} ;$ GPNAase Activity=I.U.; Age =years old. 
normal controls was observed.

Table II shows serum GPNAase activity in patients with essential hypertension. Mean serum GPNAase activity in male patients with essential hypertension treated with drugs was $70.8 \pm 3.4$ I.U. and in patients without drugs was $73.1 \pm 5.0$ I.U. Serum GNPAase activity in male patients with essential hypertension was slightly elevated as compared with that of the control group $(68.8 \pm 0.27$ I.U.). But the difference was not statistically significant. In contrast, mean GPNAase activity in female patients with essential hypertension treated with drugs was $71.6 \pm 3.5 \mathrm{I}$.U. and in patients without drugs was $60.2 \pm 2.5$ I.U., and the enzyme activity in female patients with essential hypertension treated with drugs was slightly but significantly $(p<0.02)$ elevated as compared with that of the female control group (62.4 \pm 0.62 I.U.).

Table III shows serum GPNAase activity in the age-matched patients with essential hypertension. There was no significant difference between the control group and the age-matched patients with essential hypertension.

Table IV shows the distribution pattern of GPNAase activities in sera obtained from various parts of the human body by right cardiac catheterization. There were no significant differences between them.

Table III. Serum GPNAase Activity in Patients with Essential Hypertension (Age Matched) I. U.

\begin{tabular}{|c|c|c|c|c|c|c|c|}
\hline \multicolumn{2}{|c|}{ Age } & $20-29$ & $30-39$ & $40-49$ & $50-59$ & $60-69$ & $70-79$ \\
\hline \multirow{2}{*}{ Male } & Control & $71.9 \pm 0.91$ & $\begin{array}{c}69.8 \pm 0.35 \\
(617)\end{array}$ & $68.6 \pm 0.50$ & $\begin{array}{c}66.4 \pm 0.76 \\
(162)\end{array}$ & $\begin{array}{l}75.0 \\
\text { (i) }\end{array}$ & \\
\hline & E.H. & $70.5 \frac{ \pm}{(2)}$ & $\begin{array}{c}76.0 \pm 6.6 \\
(6)\end{array}$ & $\frac{68.9 \pm 4.9}{(16)}$ & $\begin{array}{c}63.3 \pm 2.0 \\
(9)\end{array}$ & $\frac{68.2 \pm 4.8}{(6)}$ & $48.5 \pm 2.1$ \\
\hline \multirow{2}{*}{ Female } & Control & $\begin{array}{c}60.2 \pm 0.78 \\
(109)\end{array}$ & $59.5 \pm 1.9$ & $64.4 \frac{4}{(20)}$ & $76.1 \pm 2.2$ & $\begin{array}{c}73.0 \\
\text { (1) }\end{array}$ & \\
\hline & E.H. & - & $\begin{array}{c}58.4 \pm 4.3 \\
(7)\end{array}$ & $\frac{68.6 \pm 5.8}{(12)}$ & $77.9 \pm 5.8$ & $65.1 \pm 2.2$ & $70.0 \pm \frac{ \pm}{(2)} 0.7$ \\
\hline
\end{tabular}

E.H. =essential hypertension, Number of patients studied in parentheses.

Table IV. GPNAase Activity in Serum Obtained from Various Parts of the Body by Right Cardiac Catheterization

\begin{tabular}{l|cccccc}
\hline \multicolumn{1}{c|}{ Location } & A & IVC & SVC & RA & RV & PA \\
GPNAase & $62.0 \pm 5.0$ & $59.0 \pm 5.0$ & $61.0 \pm 4.0$ & $64.0 \pm 5.0$ & $64.0 \pm 6.0$ & $62.0 \pm 5.0$ \\
Activity (I.U.) & $(10)$ & $(11)$ & $(13)$ & $(9)$ & $(8)$ & $(10)$ \\
Number & & & & &
\end{tabular}

$A=$ peripherial artery; IVC=inferior vena cava; $S V C=$ superior vena cava; $R V=$ right ventricle; $\mathrm{PA}=$ pulmonary artery. Number of patients in parentheses. 


\section{Discussion}

In our previous paper, we had used a manual $\operatorname{method}^{7,8)}$ for the determination of GPNAase activity. But in this paper, we have used an automatic method with use of the LKB 8600 Reactor Rate Analyzer. ${ }^{91}$ The enzyme activities obtained by each method were found to be essentially the same, and there was a close correlation between the activities determined by the manual and the automatic methods $(r=0.99)$.

The physiological function of this enzyme is not yet clear, but the substrate specificity suggests some implications of the enzyme in the degradation of peptides derived from collagen and changes in collagen metabolism during process of development and aging may influence the enzyme activity. However, when the enzyme activities were compared between the younger and older groups, the mean activities were similar..$^{5)}$

A small but significant sex difference was found in the serum enzyme activity; the activity in male serum was slightly higher than that in female serum. This difference may be influenced by female sex hormones because the difference disappears after climacterium. $\left.{ }^{5}\right)$

A highly significant elevation of the enzyme activity was found in sera of patients with hepatobiliary disease and patients with gastric cancer had a significantly lower enzyme activity than the normal subjects or patients with gastric ulcer. ${ }^{4}$

In this study we have found that serum GPNAase activities in patients with heart failure and acute myocardial infarction were significantly decreased as compared with the normal control group. All patients with congestive heart failure were treated with digitalis and diuretics and well compensated, and hemodilution in patients with congestive heart failure is not an explanation for low values of GPNAase activity. The mechanism of low values in patients with heart failure is unclear at the present time. The low value of GPNAase activity in patients with acute myocardial infarction was also shown and it had continued for 12 days. In an early stage of acute myocardial infarction, hemoconcentration was induced by a poor intake of beverage and, the low GPNAase activity at an early stage of acute myocardial infarction suggests that it can not be attributed simply to hemodilution. The mechanism of low values in acute myocardial infarction remains to be explained.

In our previous paper, ${ }^{8)}$ we reported that GPNAase activity in sera of male patients with essential hypertension was significantly higher than that of normotensive healthy subjects. But, in this study, we could demonstrate that GPNAase activity in sera only of female patients with essential hyper- 
tension treated with drugs was significantly higher than that of normotensive healthy subjects. The discrepancy between the present and previous results may be due to the difference of the population studied and this significant difference in female patients may be ascribed to their aging. There was no significant difference between serum GPNAase activities in the control group and those in age-matched patients with essential hypertension.

In summary, serum GPNAase activity in patients with acute myocardial infarction and in patients with congestive heart failure was significantly lower as compared with normal controls.

\section{REFERENGES}

1. Hopsu-Havu VK, Glenner, GG: A new dipeptide naphthlamidase hydrolyzing glycyl-prolyl- $\beta$-naphthylamide. Histochemie 7: 197, 1966

2. Nagatsu I, Nagatsu T, Glenner GG: Species differences of serum amino acid $\beta$-naphthylamidases. Enzymologia Acta Biocatalytica 34: 73, 1968

3. Nagatsu I, Nagatsu T, Yamamoto T: Hydrolysis of amino acid $\beta$-naphthylamides by aminopeptidases in human parotis saliva and human serum. Experientia 24: 347, 1968

4. Hino M, Nagatsu T, Kakumu S, Okuyama S, Yoshii Y, Nagatsu I: Glycylprolyl $\beta$-naphthlamidase activity in human serum. Clin Chim Acta 62: 5, 1975

5. Hino M, Fuyamada $H$, Hayakawa $T$, Nagatsu $T$, Oya $H$, Nakagawa $Y$, Takemoto T, Sakakibara S: X-prolyl dipeptidyl-aminopeptidase activity with $\mathrm{x}$-proline $\mathrm{p}$-nitroanilides as substrates in normal and pathological human sera. Clin Chem 22: 1256, 1976

6. Oya H, Nagatsu I, Nagatsu T: Purification and properties of glycylprolyl $\beta$-naphthylamidase in human submaxillary gland. Biophys Acta 258: 591, 1972

7. Nagatsu T, Hino M, Fuyamada $H$, Hayakawa $T$, Sakakibara S, Nakagawa $Y$, Takemoto $T$ : New chronogenic substrates for $x$-prolyl dipeptidyl-aminopeptidase. Anal Biochem 74: 466, 1976

8. Fuyamada H, Hino M, Nagatsu T, Ogawa K, Sakakibara S: Serum glycylproline p-nitroanilidase activity in human hypertension. Chinica Chimica Acta 74: 177, 1977

9. Ohta N, Miyamoto M, Naka H, Kiyose H, Sakamoto M, Sasaki T: Basic studies on the measurement of glycyl-prolyl-4-nitroanilidase (GPNAase) activity. Jap J Clin Pathol 25: 165,1977 (abstr) 